\title{
Circulating exosome-derived miR-191-5p is a novel therapeutic biomarker for radiotherapy in esophageal squamous cell carcinoma patients
}

\section{HUAN WANG}

Chiba Daigaku https://orcid.org/0000-0002-9385-4608

Masayuki Kano (D mkano@chiba-u.jp)

https://orcid.org/0000-0002-1797-5176

\section{Yasunori Matsumoto}

Chiba Daigaku

\section{Takeshi Toyozumi}

Chiba Daigaku

\section{Satoshi Endo}

Chiba Daigaku

Hiroshi Suito

Chiba Daigaku

\section{Toshiki Kamata}

Chiba Daigaku

Koichiro Okada

Chiba Daigaku

Tadashi Shiraishi

Chiba Daigaku

HongXu Liu

Liaoning Cancer Institute and Hospital

Takahiro Ryuzaki

Chiba Daigaku

\section{Soichiro Hirasawa}

Chiba Daigaku

Masaya Yokoyama

Chiba Daigaku

Kazuya Kinoshita

Chiba Daigaku

Keiko lida

Chiba Daigaku

Kentaro Murakami 
Chiba Daigaku

Haruhito Sakata

Chiba Daigaku

Hisahiro Matsubara

Chiba Daigaku

\section{Research}

Keywords: Esophageal squamous cell carcinoma, Exosome, miR-191-5p, Radioresistance, Deathassociated protein kinase 1, MAPK signaling

Posted Date: April 1st, 2020

DOI: https://doi.org/10.21203/rs.3.rs-20214/v1

License: (c) (i) This work is licensed under a Creative Commons Attribution 4.0 International License. Read Full License 


\section{Abstract}

Background: Exosomes are nano-sized extracellular vesicles and are detectable in most body fluids. Circulating exosomal microRNAs are an easily obtained, and they could be minimally invasive biomarker for cancer treatment. Esophageal squamous cell carcinoma (ESCC) is one of the most aggressive carcinomas. Radiotherapy is one of the most important treatment option for ESCC, and it would thus be extremely crucial to predict therapeutic sensitivity and the patient prognosis in advance.

Methods: A search for miRNAs with a therapeutic biomarker in ESCC was performed using the miRNA expression signatures obtained from ESCC plasma before radiotherapy. miR-191-5p was selected because it was found to be associated with the prognosis in ESCC based on the findings of previous reports. As a result, we decided to perform more studies to elucidate the significance of miR-191-5p. Gainof-function analyses were performed to evaluate the functional significance of miR-191-5p in ESCC progression. The effects of miR-191-5p on ESCC radiosensitivity were determined by cell proliferation, a clonogenic survival assay and an apoptosis assay. A gene set enrichment analysis was used to investigate the downstream signaling pathway related to the miR-191-5p functions. The 5-year progression-free survival (PFS) rate was used to directly compare the usefulness of these biomarkers for determining the patient prognosis between the miR-191-5p high expression patients and low expression patients.

Results: A subset of seven microRNAs (miR-628, miR-363, miR-191-5p, miR-185, miR-148a, miR-320d, miR-30e) was identified to be candidates of therapeutic biomarker for ESCC patients underwent radiotherapy in a global microRNA expression analysis. A high miR-191-5p expression promoted ESCC cell proliferation, invasion and migration and induced G0/G1 to S/G2M transition. miRNA-191-5p overexpression promoted cell survival and reduced cell apoptosis after irradiation. Mechanistically, miR191-5p may directly target death-associated protein kinase 1 (DAPK1) to induce radiation resistance via the MAPK-JNK pathway. The 5-year progression-free survival rate for ESCC patients who underwent radiotherapy with high circulating exosomal miR-191-5p expression was significantly lower than in those with a low expression.

Conclusion: Tumor-derived exosomal miR-191-5p is a potential non-invasive biomarker for predicting the prognosis in esophageal cancer patients after radiotherapy.

\section{Background}

Esophageal cancer is the seventh-most common cancer and the sixth leading cause of mortality worldwide [1]. Esophageal squamous cell carcinoma (ESCC) comprises over $90 \%$ of all esophageal malignancies in Asia. Although the treatment of esophageal cancer has progressed in recent years, its prognosis remains poor, with a 5-year survival rate of $<20 \%$ [2].

Exosomes are nano-sized extracellular vesicles (50-100 nm in diameter) and detectable in most body fluids, such as plasma, urine, saliva, and ascites [3]. The role of exosomes in cancer progression is 
gradually being recognized by the study of their various molecular constituents, including proteins and nucleic acids. Tumor-released exosomes have been widely reported to regulate tumor progression, invasion, metastasis, and resistance to chemotherapy [4].

MicroRNAs (miRNAs) are small non-coding RNAs that control gene expression through posttranscriptional regulation [5]. MicroRNAs exert various function by targeting different genes. They act as oncogenes or tumor suppressor genes and also play an important role in predicting the therapeutic response. Several studies have suggested that circulating exosomal microRNAs, such as miRNA-21[6] and miRNA-1246[7], may be useful as clinical diagnostic or prognostic biomarkers for esophageal cancer. However, the exosomal microRNAs from blood samples associated with therapeutic efficacy have been poorly investigated in ESCC.

Radiotherapy plays a critical role in both neoadjuvant therapy and as radical treatment itself for resectable and unresectable locally advanced esophageal cancer [2]. The identification of molecules involved in ESCC radiosensitivity would be of great significance for the treatment of ESCC. Furthermore, plasma exosome tests that can be performed through blood tests would be minimally invasive by avoiding the need for a biopsy and be extremely versatile.

In the present study, we identified miRNAs related to radiosensitivity in ESCC plasma exosomes. The usefulness of circulating exosomal miR-191-5p as a biomarker for ESCC treatment was examined.

\section{Material And Methods}

\section{Clinical plasma samples}

The plasma samples were collected from 67 patients with newly diagnosed ESCC at Chiba University Hospital (Chiba, Japan) between May 2011 to April 2017 and 6 healthy donors. Blood examinations and sampling were performed before treatment. The present study was approved by the Ethics Committee of Graduate School of Medicine, Chiba University. Written informed consent was obtained from all of the patients. The patient characteristics and inclusion criteria were described in Supplementary methods

Global MiRNA expression analyses

miRCURY LNA ${ }^{\mathrm{TM}}$ microRNA arrays were performed according to the manufacturer's protocol (Exiqon, Vedbaek, Denmark). MiRNA arrays were performed for the plasma exosomes of ESCC patients before treatment. The tumor regression grade of the primary tumor (TRG-PT) was used to evaluate the therapeutic effect of chemoradiotherapy (CRT). Patients classified as Grade 3 were considered sensitive to CRT $(n=4)$, while those classified as Grade 0 or 1a were considered resistant to CRT $(n=4)$. MiRNA arrays were performed in a cohort of eight cases to investigate the difference in the miRNA expression between CRT-sensitive and CRT-resistant patients.

Extraction of exosomes from the plasma. 
Each plasma sample was centrifuged at 2,000 $\mathrm{g}$ for $20 \mathrm{~min}$ at room temperature to remove cells and debris. The supernatant containing the partially clarified plasma was transferred to a new tube and then centrifuged at $10,000 \mathrm{~g}$ for $20 \mathrm{~min}$ at room temperature. The supernatant was then transferred to a new tube, and proteinase K (Proteinase K For Total Exosome Isolation from plasma; Invitrogen, Carlsbad, Calif., United States) was added to remove the endogenous nucleases. Exosomes were isolated using an exosome isolation reagent (Total Exosome precipitation reagent from plasma; Invitrogen) according to the manufacturer's protocol.

Transmission electron microscope (TEM) observation

TEM observation was performed using a carbon-coated copper grid (Excel support film, 200 mesh, RL26A; NISSHIN EM Co., Ltd., Tokyo, Japan) with the negative stain method. Negative staining used a $2 \%$ phosphotungstic acid solution ( $\mathrm{pH}$ 7-7.4). All sample grids were subjected to hydrophilic treatment with glow-discharged treatment before absorption using plasma etching device (SEDE-GE; Meiwafosis Co., Ltd., Tokyo, Japan). One dispersed droplet of the sample and two droplets of phosphotungstic acid solution were prepared on a parafilm consisting of $20 \mu \mathrm{l}$ each, respectively. The stain protocols were the same as the sample absorption protocols, with staining performed twice. After negative staining, the grids were left to dry in a dry box overnight. The samples were then subjected to TEM observation $(\mathrm{H}-$ 7650; Hitachi High-Technologies Corporation, Tokyo, Japan) at an acceleration voltage of $80.0 \mathrm{kV}$.

MiRNA and mRNA isolation and detection by quantitative real-time polymerase chain reaction (qRT-PCR)

Total RNA was extracted from exosomes using the Total Exosome RNA and Protein Isolation Kit (Invitrogen) according to the manufacturer's protocol. Total cellular RNA was extracted using the miRNeasy Mini Kit (QIAGEN, Hilden, Germany) according to the manufacturer's protocol.

To measure the expression of death-associated protein kinase 1 (DAPK1), cDNA was generated using a High-Capacity RNA-to-DNA ${ }^{\text {TM }}$ Kit (Thermo Fisher Scientific) according to the manufacturer's protocol. qRTPCR was performed using SsoFast ${ }^{\text {TM }}$ EvaGreen Supermix (BIO-RAD). The DAPK1-specific primers were as follows: forward, 5'-TGGATATGACAAAGACACATC-3'; reverse, 5'-CTTCATGTCCTTTGACCCAGA-3'. The data were normalized to beta-actin. The fold-changes in the expression of each gene were calculated by the comparative threshold cycle $(\mathrm{Ct})$ method using the formula $2^{-\Delta \Delta C t}[8]$.

Cell cultures and miRNA transfection

The human ESCC cell lines TE11, and KYSE-960 were used in the present study. Immortalized esophageal keratinocyte cells (R2C3) that had been established in our institute were used as a control line [12]. KYSE960 were obtained from JCRB Cell Bank. TE11 was obtained from Cell Resource Center for Biomedical Research Institute of Development, Aging and Center, Tohoku University, Japan.

The miR-191-5p mimic and negative control mimic were purchased from Thermo Fisher Scientific. The cell lines TE11 and KYSE-960 were seeded into 6-well plates $\left(2.5 \times 10^{5}\right.$ cells per well), and after $24 \mathrm{~h}$, cells 
were transfected with miR-191-5p mimic or negative control mimic using Lipofectamine ${ }^{\mathrm{TM}}$ RNAi-MAX (Invitrogen) following the manufacturer's protocol. After $48 \mathrm{~h}$, cells were harvested for further investigation.

Cell proliferation and colony formation assays

Cells were seeded onto a 96-well plate at a density of 5000 cells per well after transfection with miR-191$5 p$ mimic or negative control mimic in the cell proliferation assay. The cell proliferation was then measured using a Cell Counting Kit-8 (Dojindo Molecular Technologies, Kumamoto, Japan) every $24 \mathrm{~h}$. For irradiation, cells were irradiated every $24 \mathrm{~h}$ with 0 or $5 \mathrm{~Gy}$ X-ray irradiation after cell adherence.

Cells were seeded onto a 6-well plate at various cell densities $\left(4 \times 10^{2}-8 \times 10^{3}\right)$ per well after transfection in the colony formation assay. The plates were then irradiated with a single dose of $0,2,4,6$ or 8 Gy X-ray irradiation (MBR-1520R-3; Hitachi) at $24 \mathrm{~h}$ after seeding. Following incubation for an additional 10-14 days, the cells were fixed and stained using Diff-Quick Staining (Sysmex Corp., Hyogo, Japan). The number of colonies with $>50$ cells was counted. Plating efficiency (PE) was calculated as the number of colonies divided by the number of cells seeded. The survival fraction (SF) was calculated using the following equation:

SF = Colonies Counted / Cells Seeded $\times$ (PE / 100)

The survival curve was derived from a multi-target single-hit model:

$S F=1-1-\exp \left(-D / D_{0}\right)^{n}$,

where $D_{0}$ was defined as the dose that gave an average of one hit per target. The radiation sensitivity enhancement ratio (SER) was measured according to the multi-target single-hit model [10].

Cell invasion and migration assays

Migration and invasion were detected by the transwell assay. In a 24-well plate, $5 \times 10^{4}$ transfected cells were seeded into the upper chamber (Corning Matrigel Invasion Chamber, BD Biosciences, CA, USA) with FBS-free medium. Medium with 10\% FBS was used in the lower chamber. After incubation for $36 \mathrm{~h}$, noninvading cells were removed with a cotton swab from the upper chamber, while the cells on the lower surface were fixed and stained using Diff-Quick Staining (Sysmex Corp, Yokohama, Japan). Pictures of three random fields from triplicate wells were recorded. Migration assays were performed in the same way, except the chambers had no Matrigel coating, and the incubation time was $24 \mathrm{~h}$.

Cell cycle and cell apoptosis analyses

For the cell cycle analyses, cells were starved for $24 \mathrm{~h}$ and then harvested after transfection and washed with PBS. They were then fixed in cold $70 \%$ ethanol overnight at $-4{ }^{\circ} \mathrm{C}$ and washed with PBS, incubated with $100 \mu \mathrm{g} / \mathrm{ml} \mathrm{RNaseA} \mathrm{(Invitrogen)} \mathrm{and} 0.1 \%$ Triton for 5 min at $37^{\circ} \mathrm{C}$ to remove the RNA, and then 
incubated with $50 \mu \mathrm{g} / \mathrm{ml}$ propidium iodide $(\mathrm{Pl})$ for $30 \mathrm{~min}$ at room temperature. DNA quantification was performed using a BD FACS Canto II (BD Biosciences, CA, USA), and the results were analyzed using the ModFitLT software program (BD Biosciences, CA, USA).

For the cell apoptosis analyses, cells were irradiated with 0 or $8 \mathrm{~Gy}$ X-rays after transfection. After incubation for $24 \mathrm{~h}$, the cells were harvested and washed with PBS, resuspended with $100 \mu$ Annexin V Biding Solution, and incubated with $5 \mu$ of Annexin V FITC and $5 \mu$ of PI solution (Annexin V-FITC Apoptosis Detection Kit; Nacalai Tesque, Kyoto, Japan) at room temperature for 15 min. Finally, $400 \mu l$ of Annexin V Biding Solution was added before the analysis. The results were analyzed using the FlowJo software program (TreeStar, Ashland, Oregon, United States).

Western blot analyses

Cell lysates were obtained using RIPA buffer. Protein was quantified using the BCA protein assay (BIORAD, Hercules, CA). In brief, $40 \mu \mathrm{g}$ of protein was loaded onto a $7.5-15 \%$ polyacrylamide gel (XV PANTERA GEL; DRC Perfect NT gel System Products, Kyoto, Japan) and then transferred onto a 0.2- $\mu \mathrm{m}$ PVDF membrane (Trans-Blot Turbo ${ }^{\mathrm{TM}}$ Transfer Pack; BIO-RAD) and blocked with Tris-buffered saline (TBS)/Tween 20 with $5 \%$ non-fat milk or $3 \%$ BSA. The membrane was then incubated with the primary antibodies of CD63, CD81, DPAK1, ERK, p-ERK, JNK, p-JNK, p-38, p38, p-p38, caspase3, bcl-2 (all from Santa Cruz Biotechnology, Santa Cruz, CA, USA) and beta-actin, GAPDH (Abcam) at $4{ }^{\circ} \mathrm{C}$ overnight and with the HRP-conjugated secondary anti-mouse or rabbit antibody (Sigma, St. Louis, MO, USA) for 30 min at room temperature. Immune complexes were detected using a Chemiluminescence CCD Imaging System (AE-9300 Ez-Capture MG; ATTA CORPORATION, Tokyo, Japan).

Extraction of exosomes from the cell culture medium

A total of $5 \times 10^{6}$ cells were incubated with $10 \%$ exosome-free FBS medium for $48 \mathrm{~h}$. A total of $30 \mathrm{ml}$ medium was harvested and centrifuged at $2000 \mathrm{~g}$ for $30 \mathrm{~min}$ to remove cells and debris. Total Exosome Isolation (from cell culture media) reagent $(15 \mathrm{ml}$; Invitrogen) was then added to the samples, which were incubated at $4{ }^{\circ} \mathrm{C}$ overnight. After incubation, the samples were centrifuged at $10,000 \mathrm{~g}$ for $1 \mathrm{~h}$ at $4{ }^{\circ} \mathrm{C}$. Exosomes were then resuspended in phosphate-buffered saline (PBS) for downstream analyses.

Gene set enrichment analyses

The enrichment analysis of The Cancer Genome Atlas (TCGA) database

(https://www.cbioportal.org/datasets) was performed with GSEA v4.0.1. A total of 196 RNAseq data and miRNAseq data points from esophageal cancer patients were used to evaluate the miRNA activities in cancer transcriptomes. Data were divided into miR-191-5p high-expression and low-expression groups based on the median miR-191-5p expression. A STRING analysis (https://string-db.org/) was used to predict the functional protein association networks of DAPK1.

Tumor xenografts 
Six-week-old female BALB/c Slc-nu/nu mice had $5 \times 10^{6}$ TE11 cells injected into their back. Seven days after the injection, the tumor volume was measured by calipers, with measurements subsequently repeated every three days. The mice were randomly divided into a tumor-bearing group $(n=4)$ and a tumor-free group $(n=4)$. When the average tumor volume reached $300 \mathrm{~mm}^{3}$, mice were anaesthetized with isoflurane, and blood samples were collected by cardiac puncture. Exosomes were then extracted from the plasma for further research.

The present study was performed according to the guidelines on animal experiments and approved by the animal experiment and welfare committee at Chiba University.

Statistical analyses

The statistical analyses were performed with the SPSS 21 software program (SPSS, Chicago, IL, USA) and the GraphPad Prism 7.04 software program (GraphPad Software, Inc., La Jolla, CA, USA). The difference between two groups was analyzed using Student's t-test. Differences in the expression of miR$191-5 p$ in relation to the clinical characteristics were examined using the chi-square test. The KaplanMeier method was used to plot survival curves, and the results were compared using the log-rank test. Cox's proportional hazards regression model was used to analyze the univariate and multivariate survival. $P<0.05$ was considered significant.

\section{Results}

The global miRNA expression analysis for exosomes derived from plasma in esophageal cancer patients

The expression of a subset of 7 miRNAs (miR-628, $p=0.0023$, miR-363, $p=0.0046$, miR-191-5p, $p=$ 0.0254, miR-185, $p=0.0382$, miR-148a, $p=0.0405$, miR-320d, $p=0.0432$, miR-30e, $p=0.0446$ ) significantly differed between CRT-sensitive and CRT-resistant patients (Table 1). The expression of miR628, miR-363, miR-185, miR-148a, miR-30e showed significant downregulation in the resistant group, while the expression of miR-191-5p and miR-320d showed upregulation in the resistant group. 
Table 1

Fold Change and P-values of exosomal miRNA in CRT

Resistant group vs Sensitive group

\begin{tabular}{|llll|}
\hline miRname & fold change & p.value & Regulation \\
\hline hsa-miR-628-3p & 0.68 & 0.0023 & Down \\
\hline hsa-miR-363-3p & 0.35 & 0.0046 & Down \\
\hline hsa-miR-191-5p & 1.39 & 0.0254 & Up \\
\hline hsa-miR-185-5p & 0.53 & 0.0382 & Down \\
\hline hsa-miR-148a-3p & 0.38 & 0.0405 & Down \\
\hline hsa-miR-320d & 2.07 & 0.0432 & Up \\
\hline hsa-miR-30e-5p & 0.60 & 0.0446 & Down \\
\hline
\end{tabular}

miR-191-5p expression analysis of ESCC cells and exosomes in culture medium

The miR-191-5p expression of ESCC (TE-11, KYSE960) was slightly lower than that of normal esophageal keratinocytes (R2C3), although without significance (Fig. 1A). The miR-191-5p expression in exosomes derived from ESCC culture medium was significantly higher than that in normal esophageal keratinocytes (TE11 vs. R2C3, $p=0.0012 ; \mathrm{KYSE}-960$ vs. R2C3, $p=0.0006$ ) (Fig. 1B). Two exosome markers (CD63, CD81) were confirmed by a Western blot analysis (Fig. 1C).

Proliferation And Colony Formation In Vitro

The overexpression of miR-191-5p compared with parent cells was confirmed by qRT-PCR in TE11 and KYSE-960 cell lines after miR-191-5p transfection (Supplementary Fig. 1). As shown in Fig. 2A, the cell growth curve was significantly increased after miR-191-5p mimic transfection compared with that in control cells (TE11, $p=0.0140 ; \mathrm{KYSE}-960, p=0.0141)$. In addition, the up-regulation of miR-191-5p dramatically promoted colony formation in ESCC cells (TE11, $p=0.0027$; KYSE-960, $p=0.0044$, Fig. 2B).

Cell Invasion And Migration Of ESCC After miR-191-5p Transfection

As shown in Fig. 2C, the cell invasion and migration capacity in miR-191-5p overexpression cell lines were significantly higher than in negative controls (Invasion: TE11, mimic vs. control, $p=0.007$; KYSE-960, mimic vs. control, $p<0.0001$. Migration: TE11, mimic vs. control, $p=0.00013 ; \mathrm{KYSE}-960$, mimic vs. control, $p<0.0001)$.

Cell Cycle Analyses

Cell cycle analyses showed that the percentages of ESCC cells in S/G2M phase after miR-191-5p transfection were significantly higher than in negative controls, and the percentages of ESCC cells in the 
G0/G1 phase after miR-191-5p transfection was significantly lower than in control cells (TE11, G0/G1 vs. S/G2M, $p<0.0001 ;$ KYSE-960, G0/G1 vs. S/G2M, p< 0.0001) (Supplementary Fig. 2).

Proliferation and apoptosis assays in ESCC cells after irradiation and/or miR-191-5p transfection

Cells transfected with miR-191-5p showed a higher survival rate than the negative control group after treatment with radiotherapy (RT; TE11, mimic vs. control, $p<0.0001$; KYSE-960, mimic vs. control, $p<$ 0.0001) (Fig. 3A).

The fraction of surviving cells was calculated and compared between miR-191-5p mimic and the negative control. As shown in Fig. 3B and 3C, cells transfected with miR-191-5p exhibited higher clonogenic survival rates than the negative control under irradiation (0, 2, 4, $6 \mathrm{~Gy}, \mathrm{p}<0.05)$. In addition, the miR-191$5 p$ mimics decreased the radiosensitivity of the TE11 (SER $=0.78, p<0.0001)$ and KYSE-960 cells (SER = $0.65, p<0.0001$ ) (Fig. 3D).

As shown in the Fig. 3E and 3F, the cellular apoptosis rate of miR-191-5p -transfected TE11 and KYSE-960 cells was significantly lower than in the negative mimic-transfected cells (TE11, mimic vs. control, $p=$ 0.0001; KYSE-960, mimic vs. control, $p=0.0006$ ).

Web-based bioinformatics analysis of miR-191-5p target genes and the gene expression analysis of DAPK and MAPK pathway molecules after miR-191-5p transfection

According to the bioinformatics analysis, miR-191-5p combined with position 177-183 and 770-776 of the DAPK1 3' UTR (Fig. 4A). qRT-PCR and a Western blot analysis showed that the DAPK1 mRNA and protein expression was significantly reduced after miR-191-5p transfection (Fig. 4B and 4C).

As shown in Fig. 4D, a GSEA analysis of the TCGA database revealed that radiotherapy resistance ( $\mathrm{p}<$ $0.001 ;$ NES $=1.589)$ and apoptosis resistance $(p<0.001 ;$ NES=-1.808) genes were enriched in ESCC patients with a high miR-191-5p expression. In addition, MAPK signaling pathway genes $(p=0.004$; NES $=1.460$ ) were enriched in esophageal cancer patients with a low miR-191-5p expression. The result of a STRING analysis indicated that DAPK1 interacted with the MAPK signaling pathway and caspase 3 (Fig. 4E) [10]. The activation status of three MAPK pathways-JNK, extracellular signal-regulated protein kinase (ERK), and p38 mitogen-activated protein kinase (p38 MAPK) - were confirmed with or without miR-191-5p transfection. As the results showed (Fig. 4F), the activation of JNK was inhibited by miR-191$5 p$. The activation of ERK and p38 were not significantly different between miR-191-5p transfection and the negative control. The overexpression of miR-191-5p inhibited DAPK1 and cleaved-caspase 3 expression. Bcl-2 was up-regulated by miR-191-5p (Fig. 5C).

miR-191-5p Expression Analysis Of Tumor-derive Exosome In Vivo

Exosomes were extracted from plasma of tumor-bearing and tumor-free mice. The expression of exosomal miR-191-5p was significantly higher in the tumor-bearing group than in the tumor-free group ( $p$ $=0.0014)($ Fig. 5A). 
Exosome extraction and the exosomal miR-191-5p expression analysis of ESCC patients' plasma

Exosomes were confirmed by TEM (Fig. 5B). As shown in Fig. 5C, exosomal miR-191-5p was significantly overexpressed in esophageal cancer patients compared with healthy donors according to qRT-PCR $(p=$ 0.0481).

Overall and progression-free survival (PFS) of the ESCC patients after RT

The clinical characteristics of the patients are shown in Table 2. Patients were divided into a highexpression group $(n=33)$ and low-expression group $(n=34)$ according to the expression of miR-191-5p. The clinical characteristics did not marked differ between the two groups. Patients who underwent RT with a low miR-191-5p expression showed a longer PFS than those with a high expression ( $p=0.026$, logrank test) (Fig. 6A). The disease-specific survival rate of the low-expression group was higher than in the high-expression group, although not to a significant degree $(p=0.052$, log-rank test) (Fig. 6B). The univariate and multivariate analyses of the factors associated with the PFS of RT patients indicated that the exosomal miR-191-5p expression and cStage were independent prognostic factors (Table.3). 
Table 2

Demographics and clinicopathological characteristics in esophageal squamous cell carcinoma patients (UICC 8th) (Location: line 368)

\begin{tabular}{|c|c|c|c|}
\hline Exosomal miR-191-5p & $\begin{array}{l}\text { high } \\
(N=33)\end{array}$ & $\begin{array}{l}\text { low } \\
(N=34)\end{array}$ & $\mathbf{p}$ \\
\hline Age & $66.2 \pm 8.59$ & $66.2 \pm 8.59$ & 0.452 \\
\hline \multicolumn{4}{|l|}{ Sex,n } \\
\hline Male/ Female & $27 / 6$ & $32 / 2$ & 0.15 \\
\hline \multicolumn{4}{|l|}{ Location of the tumor } \\
\hline $\mathrm{Ce} / \mathrm{Ut} / \mathrm{Mt} / \mathrm{Lt} / \mathrm{Ae} / \mathrm{X}$ & $3 / 9 / 8 / 9 / 1 / 3$ & $2 / 6 / 15 / 10 / 1 / 0$ & 0.309 \\
\hline \multicolumn{4}{|l|}{ G category, $n$} \\
\hline G1/G2/G3/N.A & $6 / 7 / 2 / 19$ & 2/ 10/6/ 15 & 0.173 \\
\hline \multicolumn{4}{|l|}{ cT category, n } \\
\hline cT1b/ 2/ 3/4a/4b/ X & $6 / 1 / 17 / 1 / 7 / 1$ & $5 / 1 / 10 / 2 / 16 / 0$ & 0.24 \\
\hline \multicolumn{4}{|l|}{ cN category, $n$} \\
\hline $\mathrm{cNO} / 1 / 2 / 3$ & $5 / 8 / 11 / 8$ & $4 / 6 / 12 / 12$ & 0.758 \\
\hline \multicolumn{4}{|l|}{ cStage, n (\%) } \\
\hline cStage $0 / 1 / 2 / 3 / 4 a / X$ & $4 / 3 / 15 / 10 / 1$ & $3 / 2 / 9 / 20 / 0$ & 0.187 \\
\hline \multicolumn{4}{|l|}{ Treatment, n } \\
\hline Surgery after RT/CRT & 30 & 32 & 0.673 \\
\hline RT/CRT & 3 & 2 & \\
\hline \multicolumn{4}{|l|}{ pT category, n } \\
\hline pT0/ 1a/ 1b/2/3/X & $10 / 2 / 5 / 3 / 12 / 2$ & $10 / 2 / 5 / 3 / 12 / 2$ & 0.539 \\
\hline \multicolumn{4}{|l|}{ pN category, $n$} \\
\hline $\mathrm{pN} 0 / 1 / 2 / 3 / \mathrm{X}$ & $18 / 6 / 4 / 2 / 4$ & $11 / 6 / 9 / 3 / 4$ & 0.434 \\
\hline \multicolumn{4}{|l|}{ pStage, n (\%) } \\
\hline pStage TONO/ 1/ 2/ 3/ 4a/ X & $10 / 3 / 9 / 7 / 1 / 4$ & $7 / 1 / 7 / 6 / 0 / 12$ & 0.233 \\
\hline
\end{tabular}


Table 3

Hazard ratio of clinical factors for the PFS of esophageal cancer (Cox's regression hazard model)

\begin{tabular}{|llll|}
\hline Factor & p-value(univariate) & p-value(multivariate) & Hazard radio(95\%Cl) \\
\hline Age $\geq 68$ & 0.845 & 0.743 & \\
\hline Sex & 0.397 & 0.484 & \\
\hline Pathological type(poor, SCC) & 0.891 & & \\
\hline Location (Thoracic) & 0.386 & & $8.974(1.191-67.593)$ \\
\hline cStage (-cStage II) & 0.043 & 0.033 & $2.929(1.296-6.922)$ \\
\hline Exosomal miR-191-5p high & 0.022 & 0.01 & \\
\hline
\end{tabular}

\section{Discussion}

Recently, accumulating evidence has suggested that exosomes mediate inter-cell communication mechanisms, especially tumor-derived exosomes carrying different types of oncogenes secreted by the tumor cell, to promote cancer progression, metastasis and radioresistance [11].

Radiotherapy (RT) is commonly used as treatment method in different types of cancer. It can minimize the tumor size and provide an opportunity for surgical resection in advanced cancer patients. Irradiation directly damages the DNA and destroys the structure of DNA, such as by inducing single-strand breaks (SSBs) or double-strand breaks (DSBs) of DNA and cross-linking DNA to DNA, resulting in the termination of cell division and proliferation, cell necrosis, or apoptosis [12].

Radioresistance can develop through various pathways, including cancer stem cells [13], reactive oxygen species (ROS) [14], and the tumor microenvironment (TME) [15], all of which are related to the response to the irradiation. It remains the main reason for the limited efficacy of radiotherapy in esophageal cancer patients. It would thus be extremely crucial to predict therapeutic sensitivity and the ESCC patient prognosis in advance. The present study therefore explored the molecular factors of the exosome responsible for conferring radioresistance in order to develop new strategies to increase patients' sensitivity to irradiation.

MiRNAs are widely reported to be involved in radioresistance of esophageal cancer. The upregulation of miR-624[16], miR-205[17] and miR-21[18] induces radioresistance in esophageal cancer, while that of miR-338[19], miR-301[20] and miR-381[21] has been reported to increase the radiosensitivity in esophageal cancer. This study is the first report to identify the different expression profiles of circulating exosomal miRNAs in esophageal cancer patients who were sensitive or resistant to CRT and to identify miR-191-5p as significantly overexpressed in radioresistant patients. 
Accumulating data in recent years has indicated that miR-191-5p is abnormally expressed in more than 20 different cancers and is a major player in some of these entities [22]. In hepatocellular carcinoma, for example, miR-191-5p was shown to act as a potential therapeutic target because it promoted proliferation and invasion and reduced apoptosis of cholangiocarcinoma cells through the miR-191/TET1/p53 pathway [23]. It was also reported that miR-191-5p was abnormally expressed in osteosarcoma and promoted cell proliferation, migration, and invasion through the EGR1 and PI3K/AKT pathways [24]. Furthermore, a high miR-191-5p expression has been reported to be associated with a bad prognosis in pancreatic cancer [25], AML [26], and breast cancer [27].

In the present study, miR-191-5p was confirmed to promote ESCC cell proliferation, invasion and migration and induce G0/G1 to S/G2M transition in the cell cycle. In addition, the overexpression of miR191-5p promoted the cell survival and decreased cell apoptosis after irradiation. A clonogenic survival assay indicated that miR-191-5p decreased radiosensitivity 0.78- and 0.65-fold in TE11 and KYSE-960 cells, respectively. Therefore, these results indicate that miR-191-5p synergistically elicits radiation resistance by inhibiting radiation-induced apoptosis.

DAPK1 acts as a positive mediator of apoptosis induced by many apoptotic signals, including DNAdamaging agents, different death stimuli [28]. Previous studies have shown that DAPK1 is a tumorsuppressive gene and is suppressed in a variety cancers [29]. GSEA results showed that the MAPK pathway was upregulated in the miR-191-5p low-expression group and suppressed in the miR-191-5p high-expression group. DAPK1 has also been found to be a key factor regulating the MAPK pathway [28]. In a previous study, miR-191-5p inhibited TNF-a-induced apoptosis of ovarian endometriosis and endometrioid carcinoma by targeting DAPK1[30]. DAPK1 acts as an upstream activator of JNK, which is the vital downstream signal of the MAPK pathway [31]. The inhibition of JNK activation, not ERK or p38, by miR-191-5p transfection has been confirmed in our study. Taken together, these previous findings provide important insight into the fact that miR-191-5p directly targets DAPK1 to regulate irradiationinduced apoptosis by decreasing the JNK activation. In the present study, we reported for the first time that DAPK1 act as a direct target of miR-191-5p and described its potential signaling pathway in ESCC. However, further studies will be required to obtain more evidence concerning the relationship between DAPK1 and the JNK signaling pathway.

The difference in the expression of miR-191-5p between tumor cells and tumor cell-derived exosomes was investigated in vitro in the present study. Interestingly, the miR-191-5p expression was slightly lower in cancer cells than in normal cells but was markedly higher in exosomes than in normal esophageal keratinocytes. These results suggest that miR-191-5p may be secreted into the circulation in greater amounts than is expressed in tumor cells, via exosomes. Furthermore, the plasma exosomes miR-191-5p expression may be a better reflection of a cancer-bearing patient's status than the miR-191-5p expression in tumor tissue assessed by a biopsy. However, further investigations on these points are still needed.

Patients who underwent RT with a low expression of miR-191-5p in circulating exosomes showed a longer PFS than those with a high expression of miR-191-5p. While there is no notable evidence that miR- 
191-5p affects the disease-specific survival of esophageal cancer, miR-191-5p was reported to be frequently overexpressed in ESCC tissues and significantly related to an advanced clinical stage, metastasis, and poor survival rate of ESCC [32]. Therefore, circulating exosomal miRNA-191 may act as a potential prognostic biomarker in ESCC patients after RT.

\section{Conclusion}

In conclusion, the tumor-derived exosome miR-191-5pmi expression was suggested to be a useful noninvasive biomarker for predicting the prognosis in patients with esophageal cancer after radiation therapy. In addition, the present study provides evidence that miR-191-5p directly targets DAPK1 to regulate irradiation-induced apoptosis by decreasing the JNK activation.

\section{Abbreviations}

\begin{tabular}{|ll|}
\hline Abbreviations & \\
\hline ESCC & Esophageal squamous cell carcinoma \\
\hline ROS & Reactive oxygen species \\
\hline TME & Tumor microenviroment \\
\hline TPG-PT & The tumor regression grade of the primary tumor \\
\hline CRT & Chemoradiotherapy \\
\hline qRT-PCR & Quantitative real-time polymerase chain reaction \\
\hline DAPK1 & Death-associated protein kinase 1 \\
\hline TCGA & The Cancer Genome Atlas \\
\hline UTR & Untranslated region \\
\hline MAPK & Mitogen-activated protein kinase \\
\hline GSEA & Gene set enrichment analyses \\
\hline JNK & C-Jun N-terminal kinases \\
\hline ERK & The extracellular-signal-regulated kinase \\
\hline
\end{tabular}

\section{Additional Files}

Additional file 1: Figure S1. qRT-PCR of miR-191-5p expression in TE11 and KYSE-960 transfected miR191-5p or negative control. Figure S2. miR-191-5p was confirmed to induce G0/G1 to S/G2M transition in the cell cycle. (231KB, docx) 


\section{Declarations}

\section{Author contributions}

Huan Wang, Masayuki Kano and Hisahiro Matsubara: Concept and design.

Yasunori Matsumoto, Takeshi Toyozumi, Satoshi Endo, Hiroshi Suito, Toshiki Kamata, Koichiro Okada, Tadashi Shiraishi: Acquisition of data.

Huan Wang, Hongxu Liu and Keiko lida: Methodology.

Kentaro Murakami and Haruhito Sakata: Analysis and interpretation of data.

Huan Wang, Masayuki Kano: wrote the manuscript

Takahiro Ryuzaki, Soichiro Hirasawa, Masaya Yokoyama, Kazuya Kinoshita: revised the manuscript.

All authors read and approved the final manuscript.

\section{Ethics approval and consent to participate}

The present study was approved by the Ethics Committee of Graduate School of Medicine, Chiba University. Written informed consent was obtained from all of the patients.

\section{Acknowledgments}

We are grateful to Dr. Q. Li and K. Tang for the technical advice and excellent technical assistance.

\section{Consent for publication}

All authors agree to the publication of this manuscript. This manuscript has not been published and is not under consideration for publication elsewhere.

\section{Availability of data and material}

All data generated or used in this study are included in this published article and its additional files. Further details are available on request.

\section{Declaration of competing interest}

The authors declare that there is no conflict of interest.

\section{Funding sources}

This research did not receive any specific grant from funding agencies in the public, commercial, or notfor-profit sectors. 


\section{References}

1. Bray F, Ferlay J, Soerjomataram I, Siegel RL, Torre LA, Jemal A: Global cancer statistics 2018: GLOBOCAN estimates of incidence and mortality worldwide for 36 cancers in 185 countries. CA Cancer J Clin 2018, 68(6):394-424.

2. Lagergren J, Smyth E, Cunningham D, Lagergren P: Oesophageal cancer. Lancet 2017, 390(10110):2383-2396.

3. Kalluri R: The biology and function of exosomes in cancer. J Clin Invest 2016, 126(4):1208-1215.

4. Hoshino A, Costa-Silva B, Shen TL, Rodrigues G, Hashimoto A, Tesic Mark M et al: Tumour exosome integrins determine organotropic metastasis. Nature 2015, 527(7578):329-335.

5. Berezikov E, Guryev V, van de Belt J, Wienholds E, Plasterk RH, Cuppen E: Phylogenetic shadowing and computational identification of human microRNA genes. Cell 2005, 120(1):21-24.

6. Tanaka Y, Kamohara H, Kinoshita K, Kurashige J, Ishimoto T, Iwatsuki M et al: Clinical impact of serum exosomal microRNA-21 as a clinical biomarker in human esophageal squamous cell carcinoma. Cancer 2013, 119(6):1159-1167.

7. Takeshita N, Hoshino I, Mori M, Akutsu Y, Hanari N, Yoneyama Y et al: Serum microRNA expression profile: miR-1246 as a novel diagnostic and prognostic biomarker for oesophageal squamous cell carcinoma. Br J Cancer 2013, 108(3):644-652.

8. Livak KJ, Schmittgen TD: Analysis of relative gene expression data using real-time quantitative PCR and the 2(-Delta Delta C(T)) Method. Methods 2001, 25(4):402-408.

9. Huang Q, Zhang S, Zhang H, Han Y, Liu H, Ren F et al: Boosting the Radiosensitizing and Photothermal Performance of Cu2-xSe Nanocrystals for Synergetic Radiophotothermal Therapy of Orthotopic Breast Cancer. ACS Nano 2019, 13(2):1342-1353.

10. Szklarczyk D, Gable AL, Lyon D, Junge A, Wyder S, Huerta-Cepas J et al: STRING v11: protein-protein association networks with increased coverage, supporting functional discovery in genome-wide experimental datasets. Nucleic Acids Res 2019, 47(D1):D607-D613.

11. Azmi AS, Bao B, Sarkar FH: Exosomes in cancer development, metastasis, and drug resistance: a comprehensive review. Cancer Metastasis Rev 2013, 32(3-4):623-642.

12. Wang H, Mu X, He H, Zhang XD: Cancer Radiosensitizers. Trends Pharmacol Sci 2018, 39(1):24-48.

13. Rycaj K, Tang DG: Cancer stem cells and radioresistance. Int J Radiat Biol 2014, 90(8):615-621.

14. Wang H, Jiang H, Van De Gucht M, De Ridder M: Hypoxic Radioresistance: Can ROS Be the Key to Overcome It? Cancers (Basel) 2019, 11(1).

15. Barker HE, Paget JT, Khan AA, Harrington KJ: The tumour microenvironment after radiotherapy: mechanisms of resistance and recurrence. Nat Rev Cancer 2015, 15(7):409-425.

16. He Y, Mingyan E, Wang C, Liu G, Shi M, Liu S: CircVRK1 regulates tumor progression and radioresistance in esophageal squamous cell carcinoma by regulating miR-624-3p/PTEN/PI3K/AKT signaling pathway. Int J Biol Macromol 2019, 125:116-123. 
17. Pan F, Mao H, Bu F, Tong X, Li J, Zhang S et al: Sp1-mediated transcriptional activation of miR-205 promotes radioresistance in esophageal squamous cell carcinoma. Oncotarget 2017, 8(4):57355752.

18. Huang S, Li XQ, Chen X, Che SM, Chen W, Zhang XZ: Inhibition of microRNA-21 increases radiosensitivity of esophageal cancer cells through phosphatase and tensin homolog deleted on chromosome 10 activation. Dis Esophagus 2013, 26(8):823-831.

19. Park M, Yoon HJ, Kang MC, Kwon J, Lee HW: MiR-338-5p enhances the radiosensitivity of esophageal squamous cell carcinoma by inducing apoptosis through targeting survivin. Sci Rep 2017, 7(1):10932.

20. Su H, Wu Y, Fang Y, Shen L, Fei Z, Xie C et al: MicroRNA301a targets WNT1 to suppress cell proliferation and migration and enhance radiosensitivity in esophageal cancer cells. Oncol Rep 2019, 41(1):599-607.

21. Zhou S, Ye W, Ren J, Shao Q, Qi Y, Liang J et al: MicroRNA-381 increases radiosensitivity in esophageal squamous cell carcinoma. Am J Cancer Res 2015, 5(1):267-277.

22. Nagpal N, Kulshreshtha R: miR-191: an emerging player in disease biology. Front Genet 2014, 5:99.

23. Li H, Zhou ZQ, Yang ZR, Tong DN, Guan J, Shi BJ et al: MicroRNA-191 acts as a tumor promoter by modulating the TET1-p53 pathway in intrahepatic cholangiocarcinoma. Hepatology 2017, 66(1):136151.

24. Chen B, Zheng ZY, Yang JZ, Li XG: MicroRNA-191-5p promotes the development of osteosarcoma via targeting EGR1 and activating the PI3K/AKT signaling pathway. Eur Rev Med Pharmacol Sci 2019, 23(9):3611-3620.

25. Song Z, Ren H, Gao S, Zhao X, Zhang H, Hao J: The clinical significance and regulation mechanism of hypoxia-inducible factor-1 and miR-191 expression in pancreatic cancer. Tumour Biol 2014, 35(11):11319-11328.

26. Garzon R, Volinia S, Liu CG, Fernandez-Cymering C, Palumbo T, Pichiorri F et al: MicroRNA signatures associated with cytogenetics and prognosis in acute myeloid leukemia. Blood 2008, 111(6):31833189.

27. Nagpal N, Ahmad HM, Molparia B, Kulshreshtha R: MicroRNA-191, an estrogen-responsive microRNA, functions as an oncogenic regulator in human breast cancer. Carcinogenesis 2013, 34(8):1889-1899.

28. Elbadawy M, Usui T, Yamawaki H, Sasaki K: Novel Functions of Death-Associated Protein Kinases through Mitogen-Activated Protein Kinase-Related Signals. Int J Mol Sci 2018, 19(10).

29. Bialik S, Kimchi A: The death-associated protein kinases: structure, function, and beyond. Annu Rev Biochem 2006, 75:189-210.

30. Tian X, Xu L, Wang P: MiR-191 inhibits TNF-alpha induced apoptosis of ovarian endometriosis and endometrioid carcinoma cells by targeting DAPK1. Int J Clin Exp Pathol 2015, 8(5):4933-4942.

31. Eisenberg-Lerner A, Kimchi A: DAP kinase regulates JNK signaling by binding and activating protein kinase D under oxidative stress. Cell Death Differ 2007, 14(11):1908-1915. 
32. Gao X, Xie Z, Wang Z, Cheng K, Liang K, Song Z: Overexpression of miR-191 Predicts Poor Prognosis and Promotes Proliferation and Invasion in Esophageal Squamous Cell Carcinoma. Yonsei Med J 2017, 58(6):1101-1110.

\section{Figures}

A

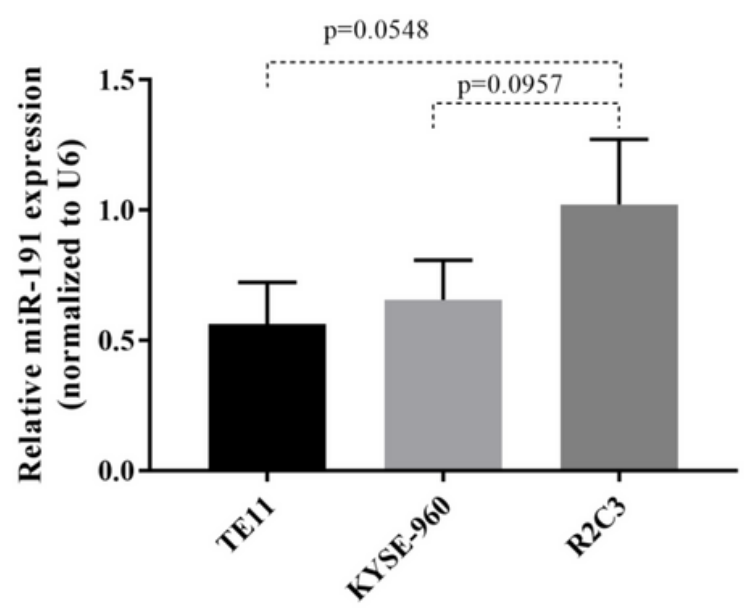

B

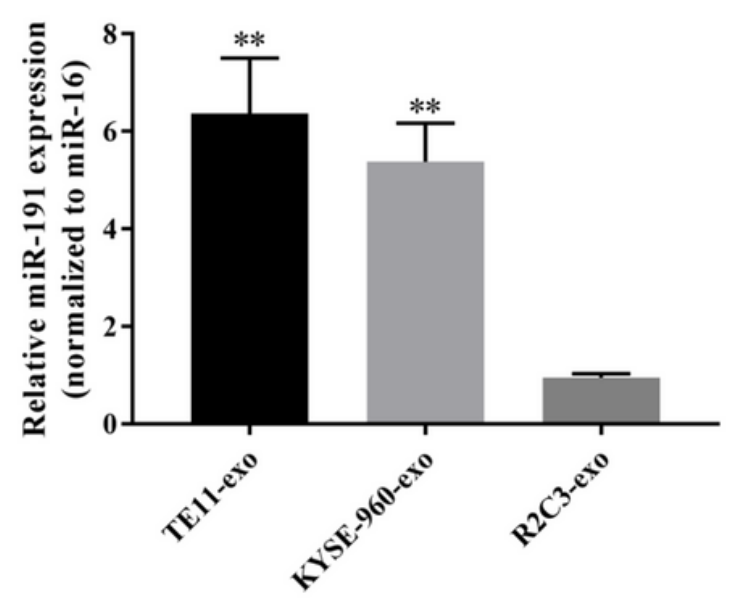

C KYSE-960 TE11 R2C3

CD63

CD81

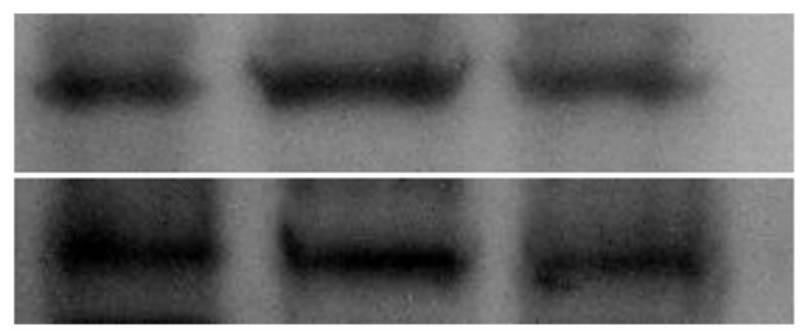

\section{Figure 1}

Results of the miR-191-5p expression analysis of ESCC cells and exosomes in culture medium. A: miR191-5p expressions of ESCC (TE-11, KYSE960) and normal esophageal keratinocytes (R2C3). B: Exosomes were extracted from cell culture medium using exosome-free medium. The miR-191-5p expression was determined by RT-PCR C: Exosome markers (CD63, CD81) were used to identify exosomes. TE11 and KYSE-960 were transfected with miR-191-5p mimic and negative control mimic for $48 \mathrm{~h}$. 
A
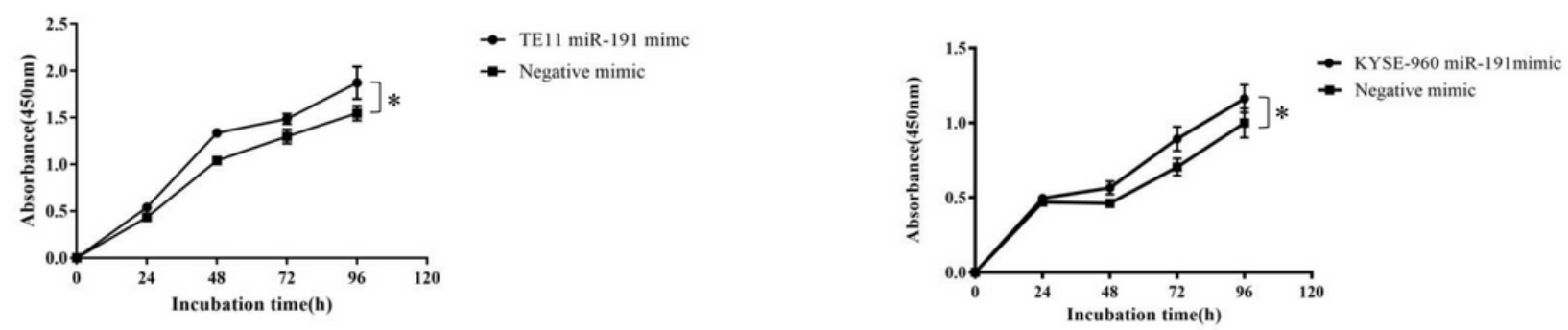

B
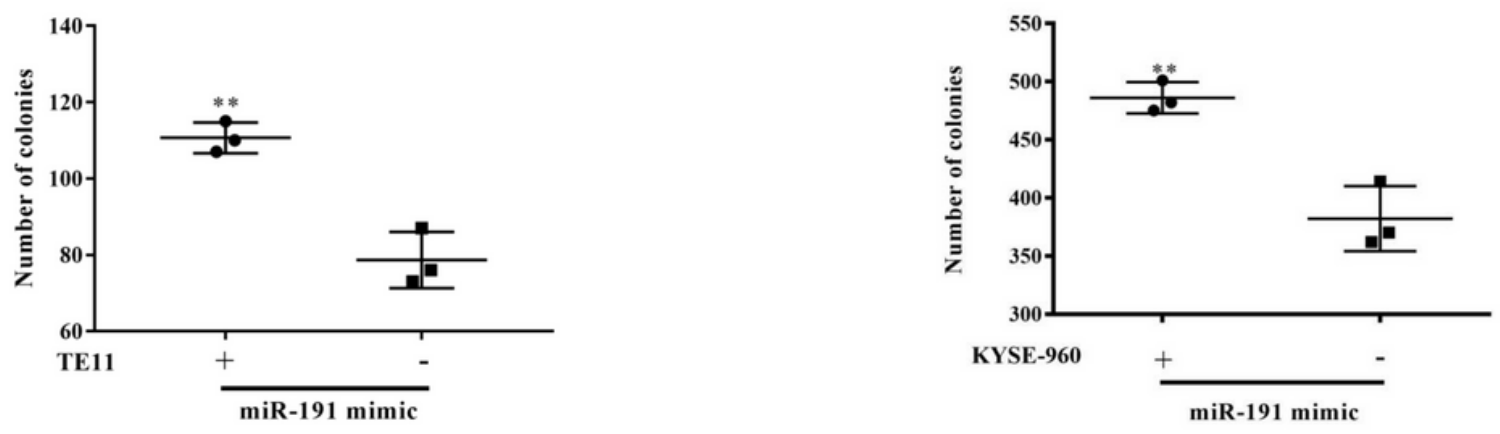

C
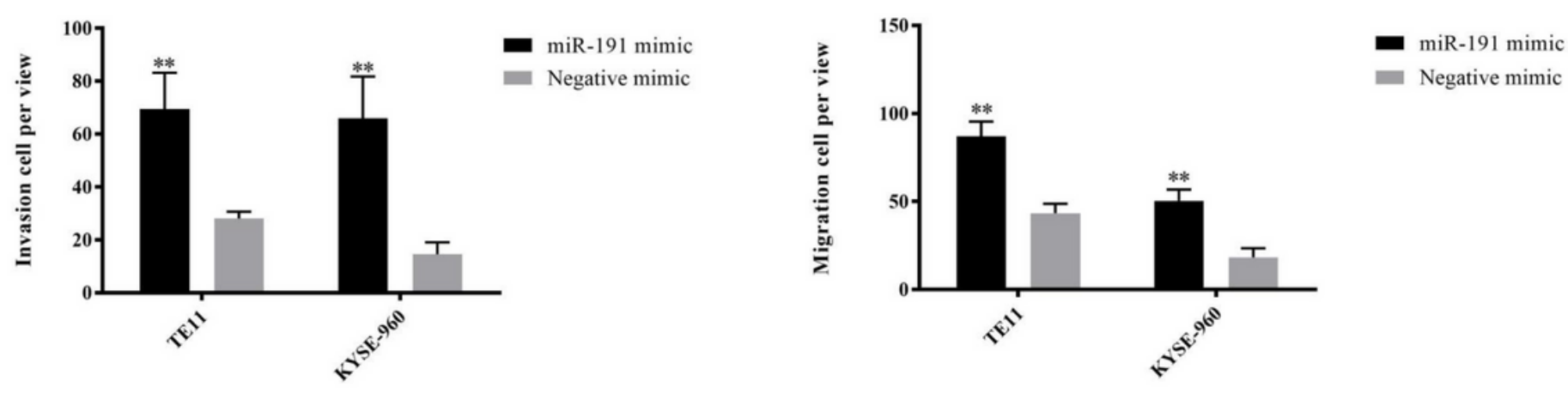

Figure 2

The overexpression of miR-191-5p promoted ESCC proliferation, colony formation, cell invasion and migration. A: The effect of miR-191-5p transfection on ESCC cells proliferation were measured by Cell Counting Kit-8. B: Colony formation assay of TE11 and KYSE-960. The cells were seeded in a 6-well plate at a density of 800 cells per well after transfection. After 10 days, the cells were stained using Diff-Quick Staining. The data are presented as the means \pm SEM. C: Migration and invasion were detected by transwell assays. A total of $5 \times 104$ transfected cells were seeded into the upper chamber with FBS-free medium, while medium with $10 \%$ FBS was used in the lower chamber. After incubation for 24 (migration assay) or $36 \mathrm{~h}$ (invasion assay), the cells on the lower surface were fixed and stained. The data are presented as the means \pm SEM. ${ }^{*} \mathrm{P}<0.05$ and ${ }^{*} \mathrm{P}<0.01$ compared with the negative control. 
A
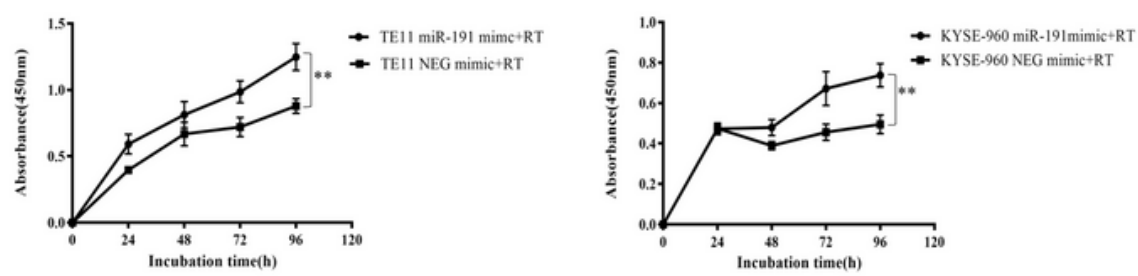

B

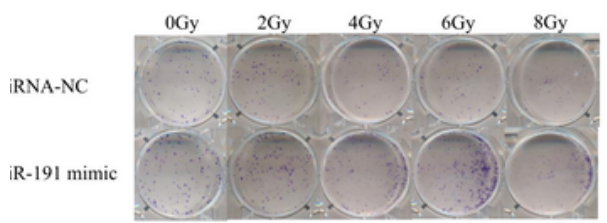

C

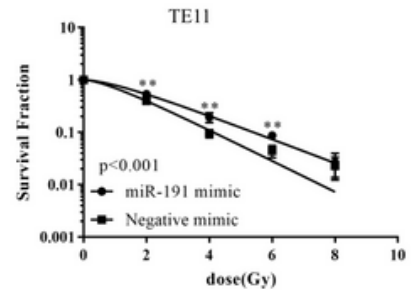

D

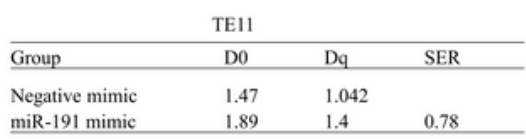

E

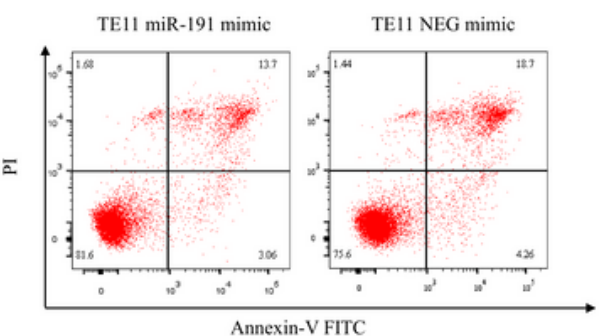

$\mathbf{F}$

KYSE-960 miR-191 mimic KYSE-960 NEG mimic

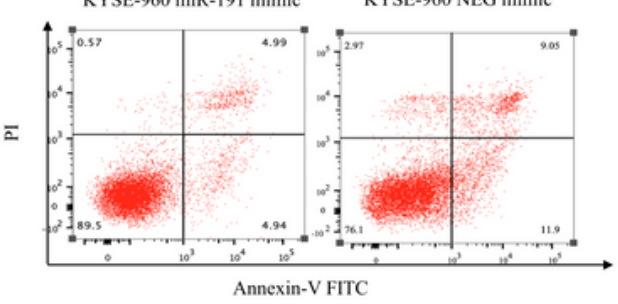

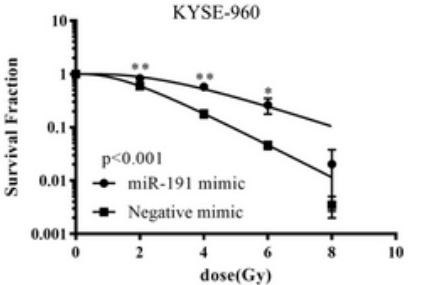

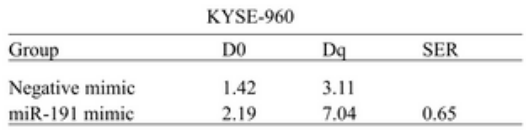

TE11

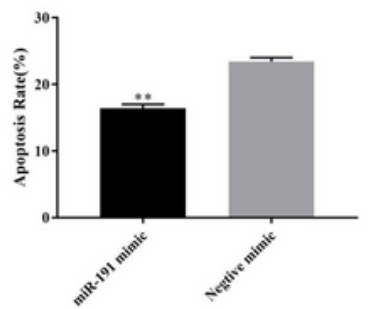

KYSE-960

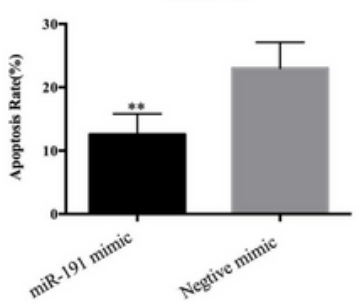

\section{Figure 3}

Proliferation and apoptosis assays in ESCC cells after irradiation and/or miR-191-5p transfection. A: After $24 \mathrm{~h}$ of cell adhesion, cells that had been transfected with mimic and mimic control were treated with 0 or $8 \mathrm{~Gy}$ of X-ray irradiation. The proliferation rate was then measured by CCK-8 every $24 \mathrm{~h}$. Clonogenic survival assays after irradiation with or without miR-191-5p transfection. B: Colony formation assays. The plates were irradiated with a single dose of $0,2,4,6$ or 8 Gy X-ray irradiation. C: The surviving 
fraction was fitted to the multi-target single-hit model. D: D0: 37\% dose slope; Dq: quasithreshold dose; SER: radiation sensitivity enhancement ratio. E, F: Annexin V/PI double-staining assays were performed to evaluate the cellular apoptosis. ${ }^{*} \mathrm{P}<0.05$ and $* * \mathrm{P}<0.01$ compared with the negative control.

A

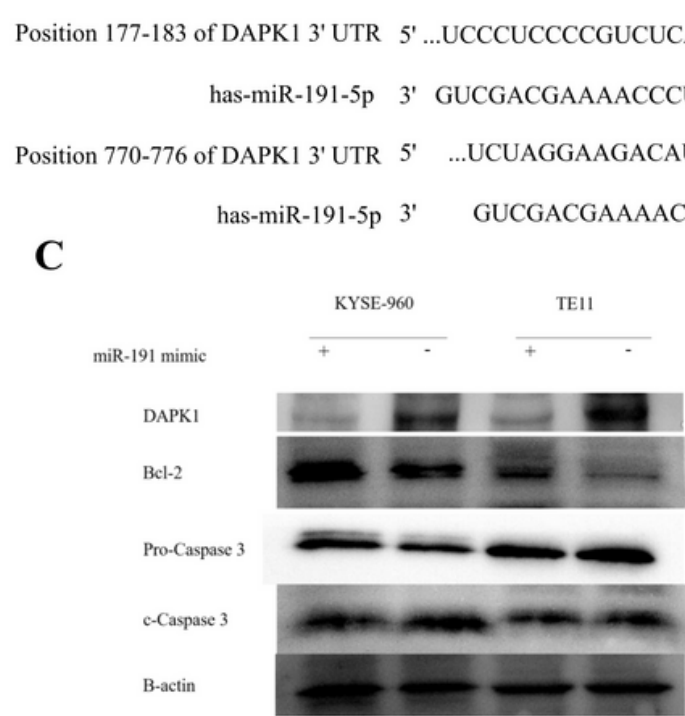

$\mathbf{E}$

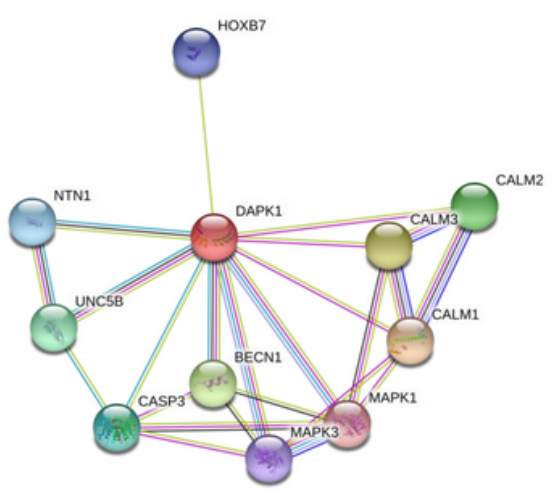

B

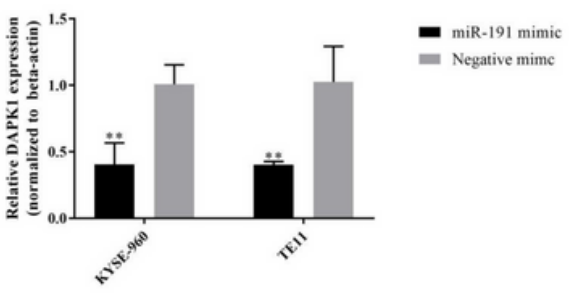

D

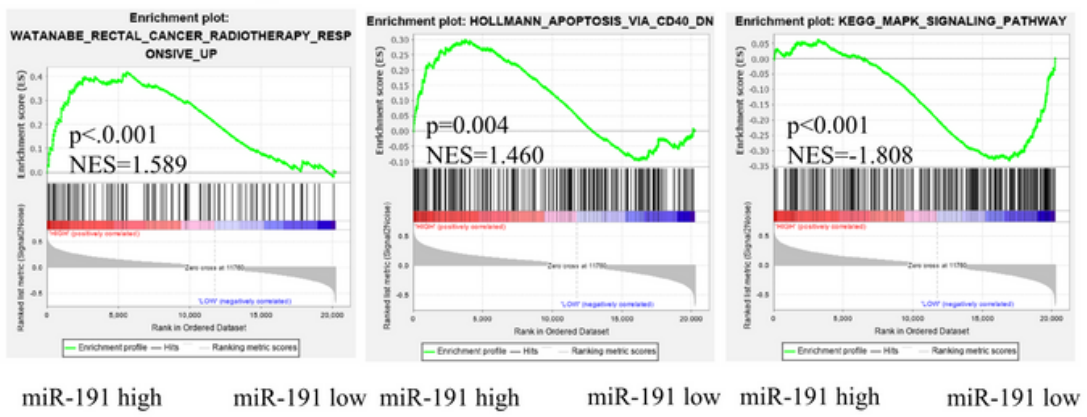

$\mathbf{F}$

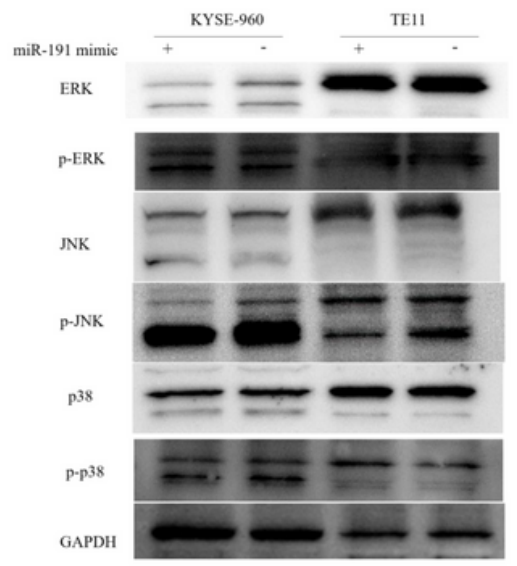

Figure 4

miR-191-5p directly targets DAPK1 to regulate apoptosis through the MAPK pathway. A. Predicted binding sites of miR-191-5p and DAPK1. B. DAPK1 mRNA expression with or without miR-191-5p transfection. C. Western blot analyses of DAPK1 and apoptotic molecules, including Bcl-2 and caspase 3, in TE11 and KYSE-960 cell lines were normalized to beta-actin. D. The enrichment analysis of The Cancer Genome Atlas (TCGA) database were performed to evaluate the miRNA activities in cancer transcriptomes. E. A STRING analysis was used to predict the functional protein association networks of DAPK1. F. Western blot analysis of the phospho-active forms of MAPKs (ERK, JNK and p38) in the TE11 and KYSE-960 cell lines were normalized to GAPDH. 


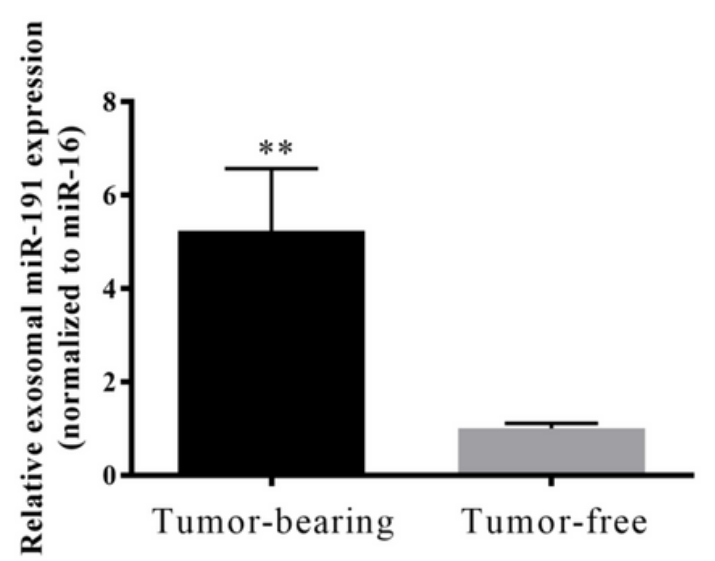

B
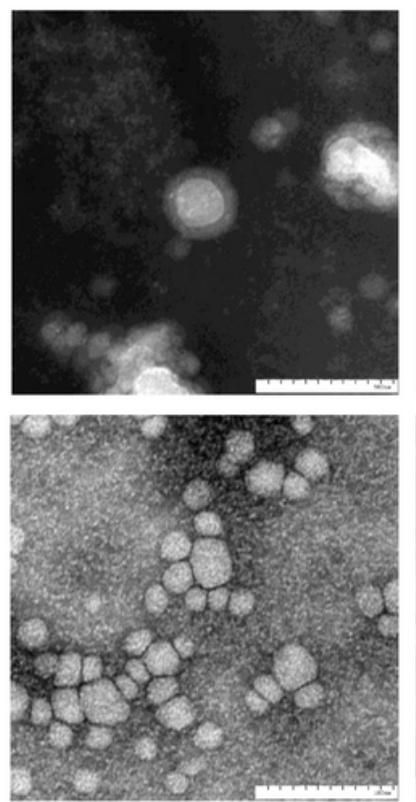
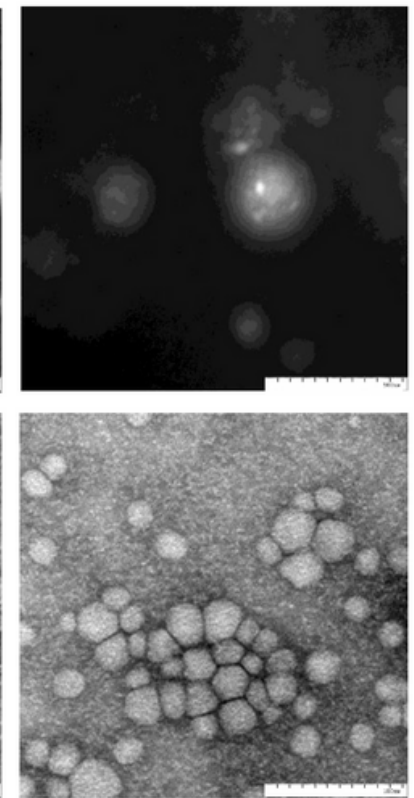

C

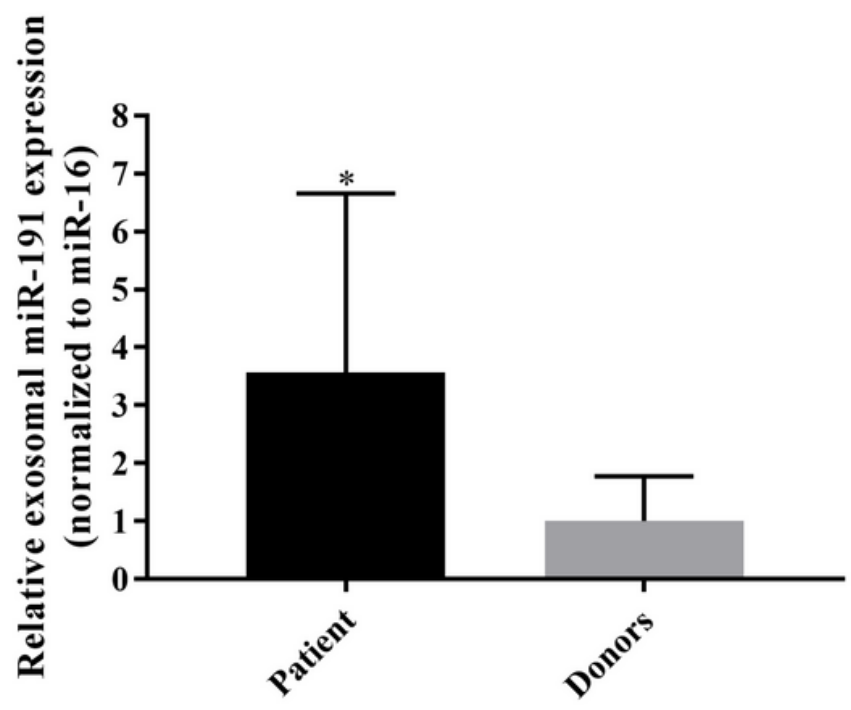

Figure 5

miR-191-5p was upregulated in the tumor-derived exosomes in vivo. Mice were divided into tumor-bearing group $(n=4)$ and tumor-free group $(n=4)$, randomly. When the average tumor volume reached $300 \mathrm{~mm}$, blood samples were collected by cardiac puncture. A: The miR-191-5p expression of the plasma exosomes was determined by PCR. B: An electron microscope image of exosome isolated from the plasma of an esophageal cancer patient. Note the presence of exosomes ranging in size from 30-100 nm. C: The overexpression of miR-191-5p in exosomes isolated from the plasma of ESCC patients. The miR191-5p expression was analyzed by qRT-PCR and normalized to the miR-16 expression. ${ }^{*}, p=0.0481$ and $\star \star \mathrm{P}<0.01$. 

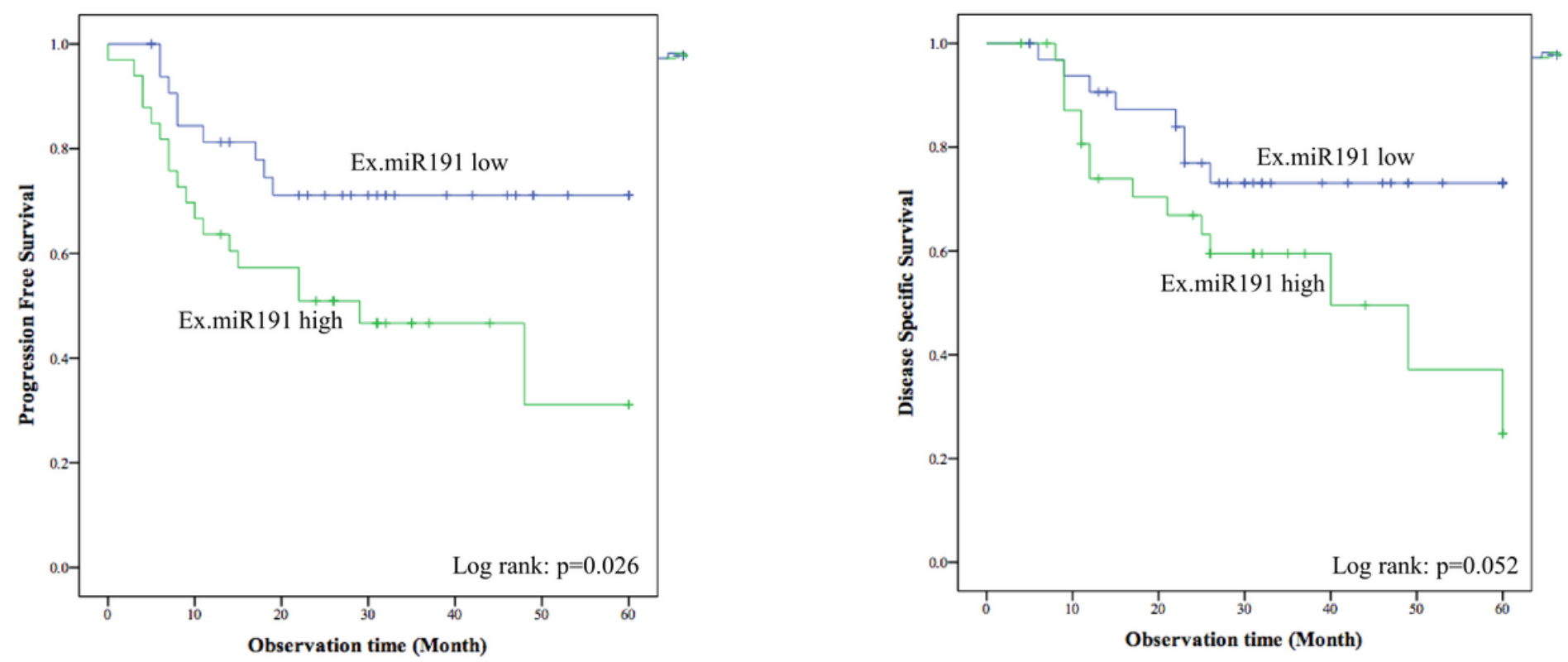

Figure 6

Kaplan-Meier analysis of the progression-free survival after radiotherapy $(A)$ and the disease-specific survival(B) was performed in 67 esophageal cancer patients according to the miR-191-5p expression. Patients who underwent RT with a low miR-191-5p expression showed a longer PFS than those with a high expression ( $p=0.026$, log-rank test).

\section{Supplementary Files}

This is a list of supplementary files associated with this preprint. Click to download.

- Additionalfile.docx 